\title{
Mise à jour d'un gène défectueux responsable de l'hémoglobinurie paroxystique nocturne
}

L'hémoglobinurie paroxystique nocturne a retenu l'attention des hématologistes depuis plus d'un siècle en raison du caractère spectaculaire des symptômes et de la nature longtemps mystérieuse du défaut cellulaire sousjacent. Il s'agit d'une anémie hémolytique très particulière : ni familiale ni congénitale, elle est caractérisée par le déficit, partiel ou total, de certaines protéines de surface érythrocytaires. Les protéines érythrocytaires, dont l'absence est responsable de l'hémolyse - le DAF (decay accelerating factor), et le CD59 ou MIRL (membrane inhibitor of reactive lysis) - protègent, chez les sujets normaux, le globule rouge contre l'action lytique du complément $[1,2]$. Chez les sujets atteints, seule une partie des globules rouges est concernée et présente une susceptibilité à l'hémolyse. Les colonies érythroïdes, obtenues après culture de cellules sanguines périphériques ou médullaires, sont, elles aussi, inhomogènes quant à l'expression de protéines de surface, de même que les colonies de cellules nucléées hématopoïétiques. L'origine monoclonale des populations cellulaires affectées a été montrée après des études d'isozymes de la glucose-6phosphate déshydrogénase comme marqueurs de l'inactivation du chromosome X [3].

Toutes les protéines manquantes partagent une propriété remarquable: elles sont attachées de façon covalente à la membrane par le même système glycolipidique, une ancre glycosaminyl-phosphatidylinositol (GPI). Plus d'une centaine de protéines exprimées à la surface des cellules eucaryotes sont attachées à la membrane par une ancre GPI. Ce n'est pas la seule fonction de l'ancre GPI : elle confère aux protéines membranaires qu'elle lie à la membrane une grande mobilité latérale ; elle sert de signal de tri pour les protéines destinées au pôle apical des cellules épithéliales, et elle est la cible de la phospholipase $\mathrm{C}$ spécifique du phosphaditylinositol qui libère, d'une part, les protéines ancrées à la membrane par son intermédiaire, et, d'autre part, le diacylglycérol, activateur de la PKC, élément important de la transmission des signaux intracellulaires (figure 1).

L'ancre GPI a une structure très conservée, indiquant une voie de biosynthèse unique. La première étape de sa synthèse est le transfert de $\mathrm{N}$ acétylglucosamine sur une molécule de phosphatidylinositol, suivie d'une désacétylation pour former le glucosaminyl-phosphatidylinositol ; trois résidus mannose sont ensuite accrochés puis une molécule de phosphoryl-éthanolamine, et le composé est alors transféré sur le carboxyl-terminal d'une protéine (figure 1). De nombreux gènes sont impliqués dans cette synthèse, dont les glycosyltransférases. La biosynthèse de l'ancre GPI a été décrite dans le cadre de l'étude de la glycoprotéine de surface variable (VSG) du trypanosome $[4,5]$ et de la glycoprotéine Thy-1 murine, toutes deux accrochées à la membrane par une ancre GPI [6]. La glycoprotéine Thy-1, protéine majeure exprimée à la surface des thymocytes et lymphocytes $\mathrm{T}$, s'est révélée un très bon modèle pour l'étude génétique somatique de l'ancre GPI ; on a établi de nombreuses lignées de lymphomes T Thy-1 $(+)$ et sélectionné des mutants Thy-1(-) n'exprimant pas en surface la protéine Thy-1. Des expériences de complémentation ont permis de les regrouper en 9 classes (AI). La base moléculaire du déficit réside pour six d'entre elles dans une anomalie post-traductionnelle affectant la synthèse de l'ancre GPI [7].
Des équipes japonaises d'Osaka, Nagoya et Fukushima ont cloné l'ADNc codant pour une protéine impliquée dans la biosynthèse de l'ancre [8]. Elles ont établi une lignée cellulaire lymphoblastoïde B humaine présentant un défaut de synthèse de l'ancre GPI, la lignée JY-5, et, par complémentation, montré qu'elle présente un défaut de classe $A$, c'est-àdire ne permettant pas les premières étapes de la synthèse de l'ancre GPI. Après transfection de ces cellules avec des $\mathrm{ADNc}$ d'une bibliothèque de cellules $\mathrm{HeLa}$ et isolement des cellules exprimant des protéines à ancrage GPI, un clone d'ADNc a été isolé qui complémentait le défaut d'expression à la surface des cellules des protéines CD59 et DAF. Il a été nommé $P I G-A$ car il code pour l'enzyme phosphatidylinositol glycane-classe A. Après confrontation aux banques de données, aucune similitude avec d'autres gènes ou protéines n'a été trouvée pour les séquences des nucléotides de l'ADN et des acides aminés de la protéine qui en découle. Cette nouvelle enzyme interviendrait dans la synthèse du $\mathrm{N}$-acétylglucosaminyl-phosphatidylinositol, l'un des premiers intermédiaires dans la synthèse de l'ancre GPI [8]. $\mathrm{L}$ 'isolement de ce gène a permis une approche génétique de l'hémoglobinurie paroxystique nocturne [9]. On a établi des lignées lymphocytaires B provenant de deux malades et présentant le déficit de protéines de surface. La transfection avec l'ADNc $P I G-A$ a restauré, dans les deux cas, l'expression des protéines déficitaires à la surface de ces cellules. Dans une lignée cellulaire affectée de l'un des malades, l'ARNm transcrit par PIG$A$ était de taille normale, mais présent en très faible quantité. La lignée affectée du deuxième malade produisait un ARNm de taille inférieure à 
la normale $(1300 \mathrm{pb}$ au lieu de 1500 $\mathrm{pb})$. L'étude des $\mathrm{ADNc}$ et génomique a permis de montrer que la délétion de $207 \mathrm{pb}$ de l' ADNc correspondait à un exon, et était due à la délétion d'une seule base dans la séquence consensus 5' d'épissage, entraînant l'excision anormale d'un exon (exon skipping). Elle a montré aussi la présence au niveau génomique de deux allèles du gène, un normal et un comportant la délétion. Pourtant aucun transcrit de taille normale n'a pu être mis en évidence dans la lignée cellulaire ; le fait que l'expression du gène anormal soit dominante a fait rechercher la localisation chromosomique de ce gène. Il s'agit du locus Xp22.1. La malade étant une femme, le gène normal réside donc probablement sur le chromosome $\mathrm{X}$ inactif dans la population cellulaire anormale. Cette localisation sur le chromosome $\mathrm{X}$ explique de plus l'expression dominante de cette mutation somatique " perte de la fonction ". L'épissage aberrant du transcrit n'entraîne pas de rupture du cadre de lecture mais la protéine issue du gène doit avoir une délétion de 69 acides aminés dans sa partie médiane. L'ADNc anormal, transfecté dans la lignée cellulaire affectée du malade, ne complémente pas l'expression de surface de la protéine CD59. Le gène anormal a été retrouvé aussi dans un grand nombre de polynucléaires et de cellules mononucléées du sang périphérique du malade, montrant que les cellules affectées provenaient du même clone de cellules souches hématopoḯtiques pluripotentes.

Cette première description d'un défaut génétique responsable de l'hémoglobinurie paroxystique nocturne confirme la plupart des hypothèses formulées jusqu'à présent. L'hémoglobinurie paroxystique nocturne n'a cependant pas encore livré tous ses secrets et, en particulier, on ne sait toujours pas pourquoi un clone défectueux et sans malignité en vient à représenter une proportion importante de la population érythroïde. L'hypersensibilité à l'activation du complément devrait être un désavantage, et pourtant il prolifère de façon importante et parfois exclusive. Le clone muté peut, en fait, avoir un avantage relatif par rapport aux autres clones s'il est capable d'échapper à un danger menaçant la population normale. On connaît la fréquence des anomalies hématologiques associées à l'hémoglobinurie

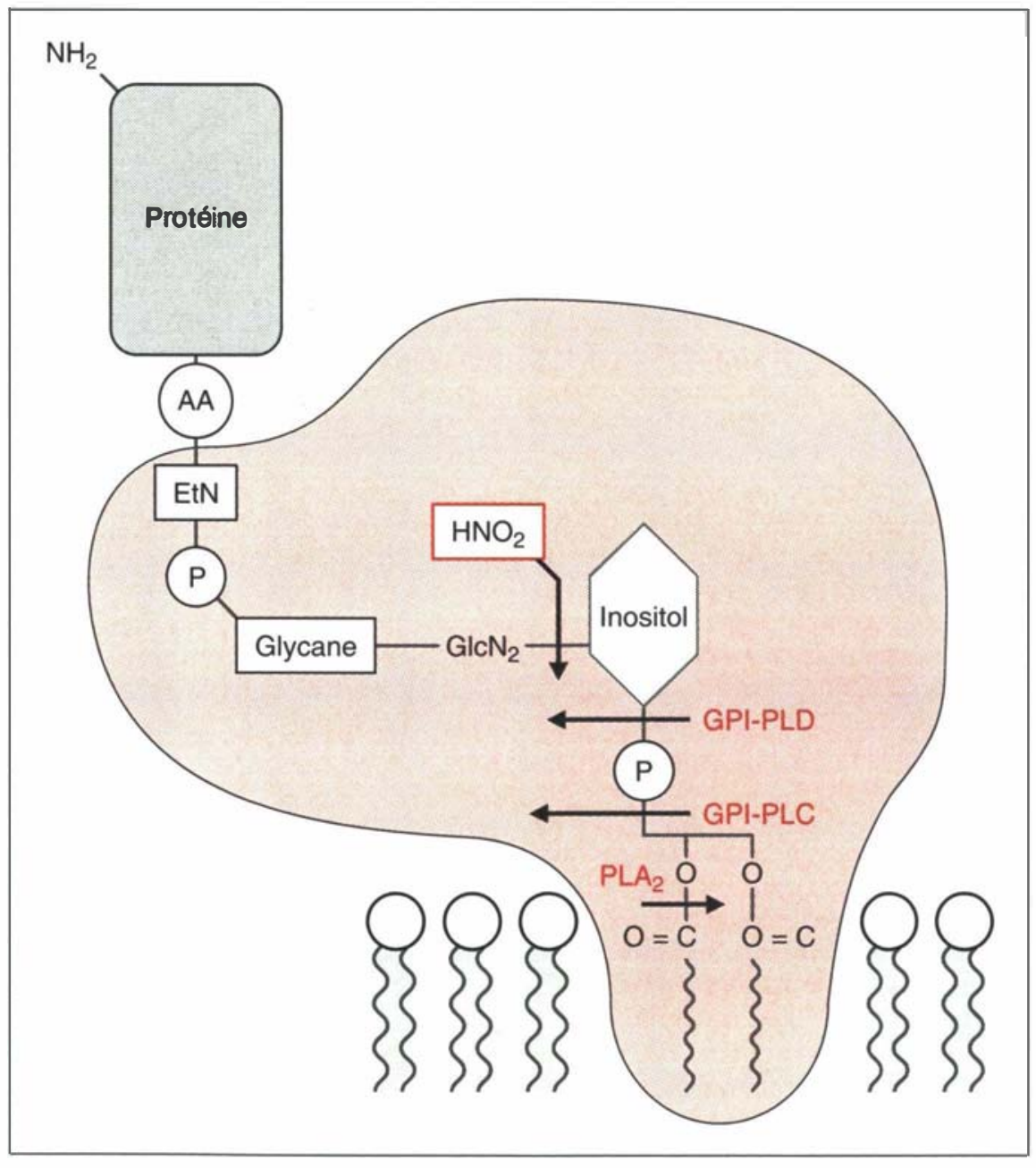

$\mathrm{m} / \mathrm{s} n^{\circ} 10 \mathrm{vol} .9$, octobre 93
Figure 1. Structure des ancres GPI. $A A$ : acide aminé $C$-terminal; EtN : éthanolamine; $P$ : phosphate. Les flèches indiquent les sites de clivage: HNO2: acide nitreux; GPI-PLD : phospholipase $D$ spécifique de l'ancre GPI : GPI-PLC : phospholipase C spécifique de l'ancre GPI ; PLA2 : phospholipase A2.

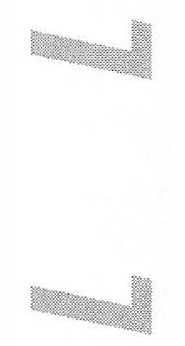


paroxystique nocturne, en particulier l'aplasie médullaire. Si l'agent délétère pour la population hématopoïétique normale (quel qu'il soit) agit par l'intermédiaire d'une protéine à ancrage GPI, la survenue d'une mutation inhibant la synthèse de l'ancre dans une cellule souche pluripotente lui permettrait d'échapper au danger encouru par les cellules normales, déterminant ainsi son expansion clonale [10].

\section{E. B.}

1. Nicholson-Weller A, March SP, Rosenfeld SI, Austen KF. Affected erythrocytes of patients with paroxysmal nocturnal hemoglobinuria are deficient in the complement regulatory protein, decay accelerating factor. Proc Nall Acad Si USA 1983 ; 80 : 5066-70.

2. Holguin $\mathrm{MH}$, Wilcox LA, Bernshaw $\mathrm{NJ}$, Rosse WF, Parker CJ. Relationship between the membrane inhibitor of reactive lysis and the erythrocyte phenotypes of paroxysmal nocturnal hemoglobinuria. J Clin Invest $1989 ; 84$ : 1387-94.

3. Oni SB, Osunkoya BO, Luzzatto L. Paroxysmal nocturnal hemoglobinuria : evidence for monoclonal origin of abnormal red cells. Blood 1970 ; 36 : 145-52.

4. Braun Breton C, Pereira da Silva L.H Modulation de l'activité biologique des protéines de surface chez les protozoaires hémoparasites. médecine/sciences $1989 ; 5: 736-43$.

5. Ferguson MAJ, Low MG, Cross GAM . Glycosyl-sn-1,2-dimyristylphosphatidylinositol is covalently linked to Trypanosoma brucei variant surface glycoprotein. J Biol Chem 1985; 260 : 14547-55

6. Homans SW, Ferguson MAJ, Dwek RA, Rademacher $\mathrm{TW}$, Anand $\mathrm{R}$, Williams $\mathrm{AF}$. Complete structure of the glycosyl phosphatidylinositol membrane anchor of rat brain Thy-1 glycoprotein. Nature $1988 ; 333$ : 269-72.

7. Hyman R. Cell-surface-antigen mutants of haematopoietic cells. Tools to study differentiation, biosynthesis and function. Biochem J $1985 ; 225: 27-40$.

8. Miyata T, Takeda J, Iida Y, Yamada N, Inoue N, Takahashi M, Maeda K, Kitani T, Kinoshita T. The cloning of PIG-A, a component in the early step of GPI-anchor biosynthesis. Science 1993; 259 : 1318-20.

9. Takeda, J Miyata T, Kawagoe K, lida Y, Endo Y, Fujita T, Takahashi M, Kitani T, Kinoshita T. Deficiency of the GPI anchor caused by a somatic mutation of the PIG-A gene in paroxysmal nocturnal hemoglobinuria. Cell 1993; 73: 703-11.

10. Rotoli B, Luzzatto L. Paroxysmal nocturnal hemoglobinuria. Semin Hematol $1989 ; 26$ : 201-7.
Un Un liposarcome myxoïde avec translocation chromosomique et production d'un facteur de transcription hybride. Très récemment, l'équipe de G. Thomas a rapporté le clonage du gène hybride engendré par la translocation caractéristique du sarcome d'Ewing ( $\mathrm{m} / \mathrm{s} n^{\circ} 9$, vol. 8, p. 994). Dans ce cas, la translocation $\mathrm{t}(11 ; 22)$ entraîne l'apparition d'un gène, d'un transcrit et d'une protéine hybrides associant des séquences correspondant à un facteur de transcription de la famille ets et à une protéine se liant à l'ARN, dénommée EWS. Anne Crozat et al., avec une équipe de New York (USA) et Lund (Suède) [1], viennent maintenant de décrire une protéine hybride semblable résultant d'une translocation $\mathrm{t}(12 ; 16)(\mathrm{q} 13 ; \mathrm{p} 11)$. Ce réarrangement chromosomique aboutit à la formation d'un gène hybride commandant la synthèse d'une protéine dont la partie aminoterminale appartient à un gène TLS et la partie carboxyterminale au gène CHOP. Le gène CHOP code pour un facteur de transcription possédant un domaine de dimérisation à glissière de leucine (leucine zipper), de la même famille que $\mathrm{C} / \mathrm{EBP}$, qui est exprimé au cours de la lipogenèse et est induit dans différentes conditions aboutissant à des altérations de l'ADN. La protéine CHOP provoquerait un arrêt des divisions, mis à profit pour réparer les altérations de l'ADN. Elle pourrait également jouer un rôle dans la régulation du cycle cellulaire au cours de la lipogenèse. La séquence TLS présente de nombreuses similitudes avec celle du gène $E W S$ réarrangé avec le gène $F L I-1$ à la suite de la translocation $\mathrm{t}(11 ; 22)$ caractéristique du sarcome d'Ewing. La protéine hybride possède le domaine de liaison à l'ADN de CHOP et le domaine effecteur présomptif de TLS, avec une région riche en glutamine et en glycine. De telles régions riches en glutamine font partie de domaines d'activation transcriptionnelle de certains facteurs de transcription, par exemple Sp1. La stimulation anormale de la prolifération pourrait donc être le résultat du remplacement du domaine effecteur de CHOP par celui de TLS. Cette protéine hybride pourrait ainsi moduler de façon anormale les gènes cibles normaux de CHOP au cours de la lipogenèse et expliquer la formation du liposarcome myxoïde.

[1. Crozat A, et al. Nature 1993 ; $363: 640-4$.

Les fonctions non enzymatiques des enzymes. Selon la sagesse populaire des biochimistes, tout est simple : les enzymes sont des catalyseurs biologiques accélérant une réaction chimique particulière. Cependant, deux exemples récents illustrent la possibilité pour des enzymes d'avoir d'autres fonctions. Nous avons très récemment reparlé dans médecine/sciences du gène $n m-23$, codant pour l'enzyme nucléoside diphosphate kinase, dont des résultats, encore controversés, indiquent que son hyperexpression dans certaines cellules cancéreuses (mélanomes, cancers du sein) aurait en effet anti-métastatique $(\mathrm{m} / \mathrm{s}$ $n^{\circ}$ 6-7, vol. 9, p. 808). La NDP kinase est une enzyme permettant la synthèse de nucléotides et dont on comprend mal comment l'hyperexpression pourrait avoir un effet d'inhibition sur l'apparition de métastases. Postel et al. (Princeton, NJ, USA) montrent maintenant qu'une protéine pratiquement identique à $n m-23$ peut se fixer sur une région régulatrice riche en purines du gène $c-m y c$, jouant alors le rôle d'activateur transcriptionnel. La relation entre l'activation transcriptionnelle de l'oncogène $c-m y c$ et l'inhibition des métastases reste peu évidente, mais on peut imaginer que d'autres gènes pourraient être également contrôlés par le produit du gène $n m-23$. De même, l'identification du produit de ce gène à un possible facteur de transcription explique mieux que son homologue chez la drosophile (gène awd) soit un gène de développement dont la mutation entraîne des anomalies des ailes. Tout semblait égale- 
ment dit des mécanismes par lesquels l'adénosine désaminase (ADA) intervenait dans la fonction lymphocytaire. Le déficit en ADA entraîne en effet une accumulation de désoxyadénosine et de désoxyadénosine-triphosphate dont l'effet toxique semble lié à une inhibition de l'enzyme ribonucléotide réductase. Les lymphocytes déficients en ADA sont ainsi incapables de proliférer en réponse à une stimulation antigénique, ce qui entraîne un syndrome de déficience immunitaire combinée sévère. Cependant, il n'existe pas toujours de corrélation nette entre la fonction immunitaire et la concentration intralymphocytaire en désoxyadénosine. Kameoka et al. (Boston, MA, USA) montrent maintenant que l'ADA peut également s'associer à la molécule d'activation CD26 [2], jouant peut-être ainsi un rôle dans la fonction de cette protéine et, de ce fait, dans la réponse immune. Alternativement, CD26 pourrait contrôler la concentration extracellulaire en ADA, qui agirait ainsi, malgré tout, par son activité enzymatique sur l'adénosine, un important médiateur de fonctions cellulaires agissant par l'intermédiaire de récepteurs membranaires spécifiques couplés aux protéines $\mathrm{G}$.

[1. Postel EH, et al. Science 1993 ; 261 : 478-80.]

[2. Kameoka J, et al. Science 1993 ; 261: 466-9.]

Les effets hypertrophiques de l'angiotensine II sont relayés par une production autocrine d'endothéline-1 dans des cardiomyocytes de rat. L'angiotensine II et l'ET-1, deux peptides possédant des propriétés vasoconstrictrices et mitogènes (p. 1084 de ce numéro), induisent tous les deux une hypertrophie cardiaque. Ito et al. [1] viennent de démontrer que l'ET-1 intervient dans l'hypertrophie cardiaque provoquée par l'angiotensine II par un mécanisme autocrine et/ou paracrine. L'analyse de l'expression de l'ARN messager codant pour la prépro ET-1 (précur-

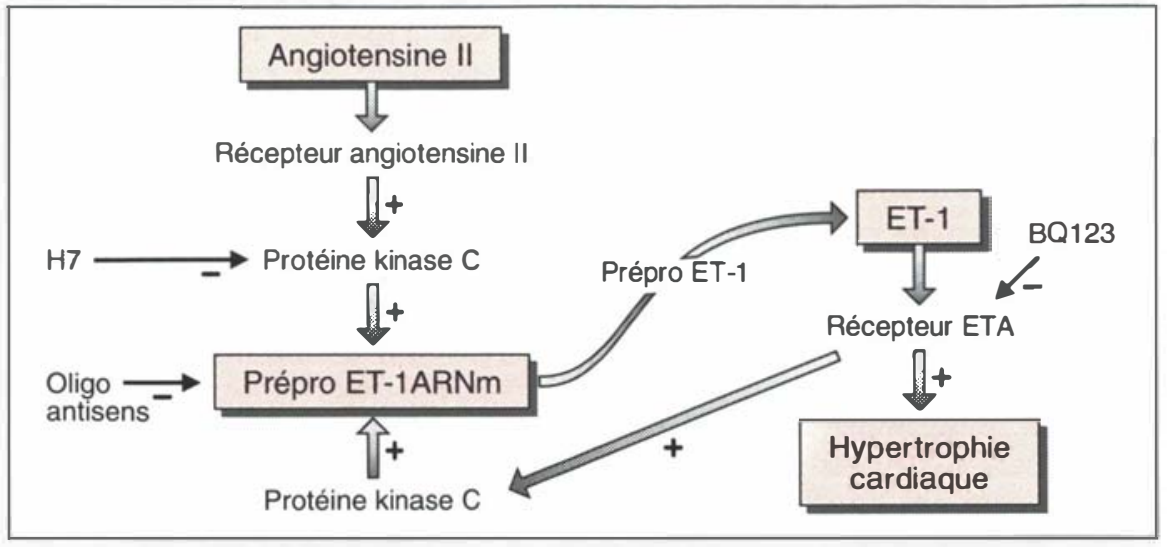

seur de l'ET-1) par Northern blot et hybridation in situ, et de la sécrétion d'ET-1 par dosage radio-immunologique, révèle que l'ET-1 est exprimée et sécrétée majoritairement par les cardiomyocytes de rats nouveau-nés et à un degré moindre par l'ensemble des autres cellules cardiaques. L'angiotensine II et l'ET-1 elle-même stimulent de 3 à 4 fois l'expression de l'ARN messager codant pour la prépro ET-1 dans les cardiomyocytes. Cette stimulation est reproduite par les esters de phorbols, et est bloquée par le $\mathrm{H} 7$, un inhibiteur de la protéine kinase C, suggérant que la PKC joue un rôle dans la régulation transcriptionnelle de l'ET-1 par l'angiotensine II et l'ET-1, en agissant probablement au niveau du TPA responsive element présent dans la région 5' du gène de l'ET-1. L'implication de l'ET-1 dans l'hypertrophie provoquée par l'angiotensine II est établie à l'aide d'une

part d'un antagoniste des récepteurs de l'ET-1 de type A, le BQ 123 (p. 1084 de ce numéro), et d'autre part d'oligonucléotides antisens complémentaires d'une partie de la région codante de la prépro ET-1, qui bloquent tous deux l'incorporation de leucine tritiée stimulée par l'angiotensine II. Les oligonucléotides antisens inhibent également l'expression de l'ARN messager de la prépro ET-1 stimulée par l'angiotensine II alors que le BQ 123 n'a aucun effet sur cette voie. Ces résultats démontrent que les effets hypertrophiques de l'angiotensine II sont relayés par une production autocrine d'ET-1 agissant par l'intermédiaire de son récepteur de type A. Ils constituent un exemple caractéristique des interactions multiples et complexes se produisant entre certains peptides vasoactifs et/ou mitogènes.

[1. Ito $\mathrm{H}$, et al. J Clin Invest 1993 ; $92: 398-403$.

\section{ACCUEIL DE JEUNES ÉQUIPES}

L'Institut de Génétique et Microbiologie de l'Université Paris-Sud (Unité de Recherche Associée au CNRS $n^{\circ}$ 1354) disposera courant 1994 de locaux pour accueillir deux chercheurs confirmés désirant constituer autour d'eux et à court terme deux nouvelles équipes. La vocation de I'IGM est I'utilisation de modèles microbiens eucaryotes et procaryotes pour l'étude de problèmes biologiques fondamentaux. Tout en souhaitant maintenir cette direction générale, I'IGM est ouvert à toute proposition de haute valeur scientifique. Les candidats doivent se manifester le plus tôt possible, surtout s'ils souhaitent poser leur candidature dans le cadre d'un programme ATIPE.

Contacter Jean-Luc Rossignol, IGM, Bâtiment 400, Université Paris-Sud, 91405 Orsay cedex, France. Tél. : 33.1.69.41.70.14. Télécopie : 33.1.69.41.66.78.
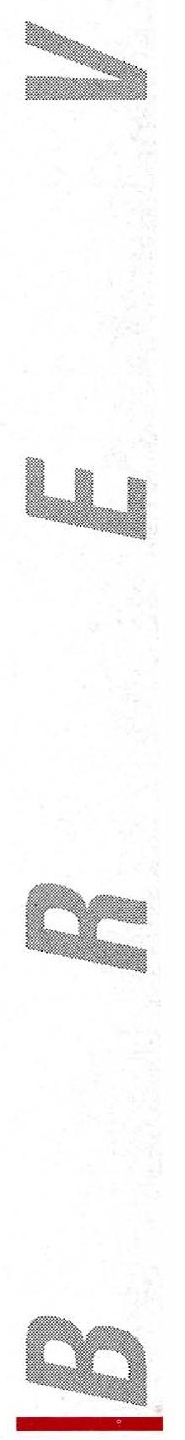